\title{
L'AUTRE EXISTE-T-IL?
}

Formulees de multiples manières, les philosophies ne posent au fond, que des questions en un nombre restreint. C'est ce qui donne son unité à la spéculation et c'est ce qui est responsable du fait qu'il y a peu de philosophies vraiment originales. On tourne autour des mêmes pots, chacun remuant la masse dedans.

Prenons l'ouvrage d'un philosophe connu seulement des milieux spécialisés, Science, Morale et Foi, de Ferdinand Gonseth, mort il y a une quinzaine d'années, et suivons le cheminement d'une pensée quasiment tourmentee par les problemes modernes de la science, sans négliger pour autant, comme l'indique le titre, d'autres champs d'investigation. Une des grandes interrogation de Gonseth concerne mon savoir au sujet des autres. En effet, l'Autre que je vois et qui pourrait bien Etre un automate, une machine - un ordinateur, dirions-nous aujourd'hui - est-il veritablement un existant comme moi? Existe-t-il pour moi, est-ce que j'existe pour lui, non seulement dans son champs visuel, mais me reconnait-il comme son semblable? Je crois me connaître assez bien, et lorsqque j'ai la sensation de la couleur rouge (par exemple), je sais ce que je ressens et comment je réagis. Cependant, lui, l'Autre, a-t-il la même sensation, la même réaction, perçoit-il et dit-il "rouge" pour les mêmes raisons que moi?

Est-ce que je sais de science sûre que l'Autre m'est analogue? Ni Descartes, ni Kant, ni Husserl, etc. n'en étaient pourt sûrs. En réalité, les philosophes de tous les temps ont postulé une inter-subjectivité qui garantirait une espèce de champs commun, un monde partage, l'accord fiable de nos aperceptions. Il s'agissait de trouver le locus ou l'agent qui nous autoriserait à dire que l'Autre pense comme moi, ce qui nous autoriserait par la suite à postuler un "inter-monde", un espace commun, une réalité extérieure à moi-même et point seulement une hallucination.

On constate bien vite que postuler une inter-subjectivite pareille revient moins à résoudre le problème de l'Autre qu'à repousser le problème lui-même plus loin. Car comment saurais-je que l'intersubjectivite, l'inter-monde, ne sont pas, eux aussi, mes présuppositions, un lieu subjectif où je suis le seul participant? Le questionnement recommence: l'inter-subjectivité est-elle réelle? Faisons remarquer que c'est là-dessus que trébuche l'esthétique transcendentale de Kant. Mes catégories mentales sont ce qu'elles sont, mon jugement ne peut sortir du 
cercle magique de l'espcace, du temps, de la causalité, tous imrprimés suigemeris dans mes structures. Ne s'agit-il pas peut-être de mon temps, de ma causalité? Mon espace est-il le même que celui de Zénon, de Descartes, d'Einstein? Mon temps et ma causalité sont-ils identique à ceux deiBergson?

$\because-$ Autrenent dit, possédons-nous une structure mentale commune, sans-laquelle l'intersubjectivité serait une mot vide, déjà vidé de sens une première fois dû au fait que le monde exterieur n'est pas démontrable. Le réel ne se prouve pas, il se constate. Il est intéressant de nous aviser que le probleme préoccupait les philosophes marxistes et autres matérialistes - voir Lénine et son ouvrage sur l'empirio-criticisme. Afin de courtcircuiter la problématique de lỉdéalisme, ils retirent el réel du cerveau (mind) et de sa structure, et le placent à plain-pied dans le monde empirique. L'épistémologie marxiste a, dès lors, la tâche facile de constater que l'esprit individuel, tous les esprits individuels, reçoivent les mêmes stimuli de l'extérieur et qu'ils les transmettent, tels quels, au cerveau. Celui-ci n'est qu'une plaque photographique; sa copie est conforme aux objets visés. Mais justement, le cerveau (mind) prend-il des photographies ou bien choisit-il son "tableau", tel le peintre à partir d'un nombre donné de couleurs qu'il arrange selon sa conception, ses préférences, son goû̀t? ${ }^{1}$

Pour les non-empiristes le problème reste entier. Voilà ce qu'en dit F. Gonseth:

\begin{abstract}
Il n'y a pas de situation pure... dans laquelle le témoin--il s'agit d'une expérimentation colorimetrique-exprimerait ce qu'il ressent dans une immediatet $\epsilon$ absolue... la participation de deux personne [appartenant au] même milieu linguistique suffit-elle pour assurer entre elles un accord intime et durable? ${ }^{2}$
\end{abstract}

L'entente sur le signaux identiques, dont le langage, ne suffit pas pour établir un discours commun, elle ne veut dire autre chose que ceci: les hommes élaborent des systèmes conventionnels qui semblent correspondre à une réalité extérieure, mais qui ne sont peut-être autre chose qu'une exigence minimale. Ce que nous appelons "subjectif" est, en

\footnotetext{
${ }^{1}$ la conclusion des penseurs marxistes est la suivante: il faut et il suffit d'amenager le monde extérieur d'une façon conforme aux desiderata sociaux pour que les hommes, tous les hommes, aient la même réaction, le mê,e comportment, les mèmes intérêts et satisfactions. Interobjectivite!

${ }^{2}$ Ferdinand Gonseth. Science, Morale et Foi. pp. 56.57.
} 
réalite, un processus complexe. Suppose qu'un autre processus complexe (un autre sujet) puisse avoir tous ses points communs avec le premier est une supposition gratuite. Gonseth appelle "reférentiel" cette quasiimperméabilité de chaque sujet. Il y a un si grand nombre d'éléments, et tellement spécifiques, qui composent mon sujet (ou mon référentiel) que je ne peux qu'ignorer si les mêmes se retrouvent chez autrui.

Citon encore Gonseth. Il y a ce qu'el appelle des essences dans ma subjectivité; elles me sont propres, et je ne puis savoir d'avance si elles jouent dans la subjectivité de tel autre le même rôle que dans la mienne... . Aucune méthode ne pourra m'assurer avec une certitude totale que sur ce point [l'énoncé d'une tâche rouge, par exemple] l'autre et moi sommes véritablement et parfaitement identiques. ${ }^{2}$

En fin de compte, cette épisté,ologie surper-critique (Hume, Russel, Gonseth, Husserl, Perelman, etc. s'enferme dans une structure subjective sans issue. L'évidence elle-même s'en trouve répudiée, pour revêtir le sens d'un enonce hypothétique à mettre à l'essai par l'intermédiaire de ses conséquences. Devant l'incompatibilité radicale des systèmes, structures et référentiels, la philosophie a le choix soit de devenir existentialiste, soit de se faire activiste. Dans les deux cas, comme le remarque C, Perelman (1970), la philosophie renonce à sa vocation de connaltre le rZel et s'efforce de guider l'action. Cependant, l'homme pose tôt ou tard la question au nom de quoi s'engage-t-il dans l'action? A l'evidence, les ideologies qui aboutissent, toutes, dans l'action, n'apportent point de réponse.

Ou plutót, il y a la réponse de F. Gonseth:

Une mutation de référentiel peut s'accompagner d'un progrès dans l'objectivité du jugement et dans la justesse des comportements. ${ }^{3}$

Enoncé difficilement justifiable car il suppose que le sujet change de référentiel en vue d'un "progrès," ce qui établit une hierarchie parmi les référentiels. Et en effet, le Gonseth vieillissant découvrit le discours moral, même s'il l'assimile encore trop à ce qu'il appelle la "socialite." Du moins admet-il, en opposition au sociologue, que la morale est davantage "que le secrétion d'une communauté qui, sans elle, ne tarderait pas à se désagréger ${ }^{\text {n4 }}$. Seulement les présupposés épistemologiques

\footnotetext{
2 Ibid, p. 63.

${ }^{3}$ Ibid, p. 126.

4 Idem, p. 142
} 
antérieurs continuent à pourchasser notre philosophe: au lieu des structures du jugement, nous parlerons de la pluralité contradictoire des systèmes de morale. Il est vrai qu'un "société ne saurait s'ordonner si personne n'y reconnaît d'obligation morale. Personne ne saurait vivie d'une existence normale dans une société sans moralitét 5 .

Faisons toutefois remarquer qu'à l'intérieur de la pensée supercritique c'est une affirmation gratuite, car le devoir moral n'y est fondé que sur un choix arbitraire. D'abord, pourquoi la société? Ensuite, pourquoi une morale plutốt qu'un multitude, à la rigueur chacune personnelle, découlant d'un référentiel donné? Et, pourquoi appeler "morale" ce qui n'est qu'un mécanisme de socialité?

Le mérite du non-aboutissementde la pensée super-critique n'est pas, de toute évidence, dans l'analyse des problemes moraux, il est de montrer la voie en direction des modeles de société. Pour cela, on quite le plan épistémologique mais grâce à cette démarche il nous est alors loisible de découvrir cette grande vérité: L'impasse épistémologique, c'est-à-dire l'impossibilité de venir au bout des rférentiels, impose un détour vers les analyses "sociales" de l'intersubjectivite. Parlons plus clair: l'impossibilité d'accorder les référentiels entre eux, nous oblige à formuler une socialité pratique. Cela, indépendamment de toute justification philosophique, bien que, de Kant a Gonseth, on postule que, soit dans la chose-en-soi, soit "dans un lieu inscrit en nous", il y a "l'obligation morale... aux conjoints de la subjectivité et de la socialitén (p. 146). Nous avons déjà dit que cette affirmation ne fournit aucune indication de ce que pourrait être la loi morale. La socialité n'esr pas la poursuite du bien.

Cependant, en ce lieu de tension, et à défaut de fonder une intersubjectivité épistémologique, on découvre les bases du contrat social en tant que substitut, transferé dans un autre domaine, de l'intersubjectivité. Il est intéressant de trouver chez les repésentants les plus prestigieux de la philosophie super-critique une espece de naiveté épistémologique, un idéalisme bon-enfant, mais qui risque de tourner en une entreprise collective éventuellement féroce.

Prenons les deux exemples déja mentionnés, Kant et Gonseth. Le premier est amené, de pas sa théorie de la connaissance bloquée devant le réel, à postuler des régimes partout républicains, une espèce de contrat universel conduisant à la paix perpétuelle. A défaut d'harmoniser les sujets au niveau des évidences, il convient d'accorder leurs travaux

\footnotetext{
3 Idem p. 143.
} 
pratiques et leur entente sur le plan social et politique. De même chez Gonseth. A l'instar de Kant, le philosophe suisse ébaucje le projet de linter-subjectivité et de la morale consensuelle chez les savants et autres chercheurs scientifiques. Ils sont, paraît-il, les mieux placés, à la fois pour se mettre d'accord sur l'objet de la connaissance et sur l'espace public moralisé. Dommage que ce consensus se revele aussi fragile que celui des non-savants.

THOMASMOLNAR 